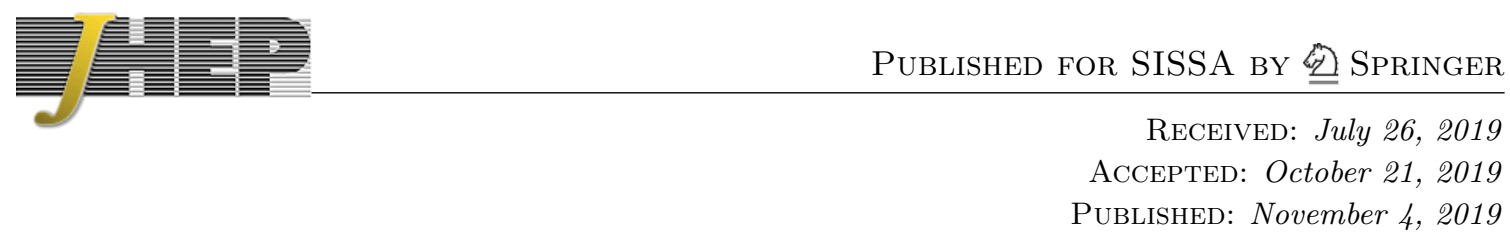

\title{
Quantum K-theory of Calabi-Yau manifolds
}

\author{
Hans Jockers ${ }^{a}$ and Peter Mayr $^{b}$ \\ ${ }^{a}$ Bethe Center for Theoretical Physics, Physikalisches Institut, Universität Bonn, \\ 53115 Bonn, Germany \\ ${ }^{b}$ Arnold Sommerfeld Center for Theoretical Physics, Ludwig-Maximilians-Universität, \\ 80333 Munich, Germany \\ E-mail: jockers@uni-bonn.de, mayr@physik.uni-muenchen.de
}

\begin{abstract}
The disk partition function of certain $3 \mathrm{~d} N=2$ supersymmetric gauge theories computes a quantum K-theoretic ring for Kähler manifolds $X$. We study the 3 d gauge theory/quantum K-theory correspondence for global and local Calabi-Yau manifolds with several Kähler moduli. We propose a multi-cover formula that relates the 3d BPS worldvolume degeneracies computed by quantum K-theory to Gopakumar-Vafa invariants.
\end{abstract}

KeYwords: Supersymmetric Gauge Theory, Chern-Simons Theories, Sigma Models ArXIV EPRINT: 1905.03548 


\section{Contents}

1 Introduction and outline 1

2 Multi-cover formula for Calabi-Yau threefolds 2

2.1 Complete toric intersections 2

2.2 Multi-cover formula for Calabi-Yau threefolds 8

$\begin{array}{llr}2.3 \text { Local conifold } & 9\end{array}$

$\begin{array}{lll}2.4 & \text { K-theoretic ring } & 13\end{array}$

3 Other dimensions $\quad 15$

\section{Introduction and outline}

The works of Nekrasov [1] and Nekrasov and Shatashvili [2, 3] establish, amongst many others, a fundamental relation between $3 \mathrm{~d}$ supersymmetric gauge theories compactified on a circle and quantum K-theory on the moduli space. For the concrete case of massless theories with a non-trivial UV-IR flow, a 3d gauge theory/quantum K-theory correspondence connecting the BPS partition functions of certain $\mathcal{N}=2$ supersymmetric gauge theories to Givental's permutation equivariant quantum K-theory [4-14] on Kähler manifold $X$ was proposed and studied in ref. [15]. It lifts the correspondence between the $\mathcal{N}=(2,2) 2 \mathrm{~d}$ gauged linear sigma model (GLSM) and quantum cohomology $[16,17]$ to $3 \mathrm{~d}$ world-volumes of topology $\Sigma \times S^{1}$, in line with the results of refs. [1, 2]. The cohomological quantum product is lifted to a K-theoretic quantum product of bundles on the moduli space of stable maps from $\Sigma$ to $X$, related to the action of Wilson line operators [18-21] in the $\mathcal{N}=2$ $3 \mathrm{~d}$ gauge theory. ${ }^{1}$ In the other direction, the $2 \mathrm{~d}$ quantum cohomology can be recovered from the small radius limit of the $3 \mathrm{~d}$ theory. It appears that the $3 \mathrm{~d}$ correspondence is more fundamental than its better known $2 \mathrm{~d}$ limit in several aspects. For example, integrality of the coefficients of the instanton expansions is manifest in $3 \mathrm{~d}$, due to their interpretation as BPS degeneracies on the world-volume, or as holomorphic Euler numbers in the quantum K-theory of refs. [4-14]. This interpretation applies also to the coefficients of the mirror map [15], giving a physical derivation of the integrality properties proven before in ref. [28] for the quintic by different methods.

In this note we continue to study the $3 \mathrm{~d}$ correspondence in the special case where $X$ is a Calabi-Yau manifold, which is the natural setup for string and M-theory. For Calabi-Yau threefolds we describe a closed formula that relates the degeneracies of $3 \mathrm{~d}$ world-volume operators to the degeneracies of BPS states in the $5 \mathrm{~d}$ target space, as counted by the Mtheoretic genus zero Gopakumar-Vafa invariants [29]. The difference between the counting

\footnotetext{
${ }^{1}$ For a discussion of quantum K-theory in the context of $\mathcal{N}=4$ supersymmetric theories see refs. [2, 3, 22-27].
} 
of BPS objects on the $3 \mathrm{~d}$ world-volume and of $5 \mathrm{~d}$ BPS objects in target space shows up in the contribution of multi-covers in $n$-pt functions with $n<3$. The presented $3 \mathrm{~d}$ worldvolume BPS counting arguments apply for higher-dimensional Calabi-Yau manifolds (and higher genera) as well, even if a M-theoretic target space interpretation is not available. From this perspective, the $3 \mathrm{~d}$ world-volume BPS degeneracies are more universal. It would be interesting to uncover the physics origin of the relationship between the $3 \mathrm{~d}$ world-volume and the target space BPS indices.

\section{Multi-cover formula for Calabi-Yau threefolds}

\subsection{Complete toric intersections}

In the following we outline the computation of quantum K-theoretic invariants for $\mathcal{N}=2$ 3d gauge theories with Higgs branches corresponding to complete intersection Calabi-Yaus (CICY) in toric hypersurfaces with several Kähler moduli. As a concrete example we consider the Calabi-Yau threefold $X=\mathbb{P}_{1,1,2,2,2}^{4}[8]$, defined as the proper transform of the zero locus of a degree eight polynomial in a smooth resolution of the weighted projective space $\mathbb{P}_{1,1,2,2,2}^{4}$. This is a Calabi-Yau hypersurface with $n_{K}=2$ Kähler moduli that has been studied in much detail in the context of $2 \mathrm{~d}$ mirror symmetry in refs. [30, 31].

Difference equations. As in ref. [15], we consider the $\mathcal{N}=2$ supersymmetric $3 \mathrm{~d}$ lift of the GLSM with gauge group $\mathrm{U}(1)^{n_{K}}$ with $n$ charged matter fields with charges $q_{a \alpha}$, representing the homogeneous coordinates of the toric ambient space $W$. In addition there are $\ell$ fields of negative charge $-d_{a \beta}$ corresponding to the hypersurface constraints of degrees $d_{a \beta}$. These data are collected in the charge vectors $l_{a}=\left(-d_{a \beta} ; q_{a \alpha}\right), a=1, \ldots n_{K}, \alpha \in N$, $\beta \in D$. The index sets $N$ and $D$ refer to the fields with Neumann and Dirichlet boundary conditions in the 3d GLSM on $S^{1} \times{ }_{q} D^{2}$. The Ward identities satisfied by the $3 \mathrm{~d}$ Wilson line operators associated with the Calabi-Yau manifold $X$ can be represented by the difference operators

$$
\begin{aligned}
\mathfrak{L}_{a}= & \prod_{\substack{\alpha \in N \\
l_{a \alpha>0}}} \prod_{j=0}^{l_{a \alpha}-1}\left(1-q^{\vartheta_{\alpha}-j}\right) \\
& -Q_{a} q^{\frac{1}{2} A_{a a}+B_{a}} q^{\sum_{i} A_{a i} \theta_{i}} \prod_{\beta \in D} \prod_{j=1}^{\left|l_{a \beta}\right|}\left(1-q^{-\vartheta_{\alpha}+j}\right) \prod_{\substack{\alpha \in N \\
l_{a \alpha}<0}} \prod_{j=0}^{\left|l_{a \alpha}\right|-1}\left(1-q^{\vartheta_{\alpha}-j}\right) .
\end{aligned}
$$

Here $A_{a b}$ and $B_{a}$ parameterize the (effective) Chern-Simons levels in the 3d gauge theory and

$$
\vartheta_{\alpha}=\sum_{a} l_{a \alpha} \theta_{a}, \quad \theta_{a}=Q_{a} \frac{\partial}{\partial Q_{a}}
$$

where $Q_{a}$ are the exponentials of the Fayet-Iliopoulos parameters. The difference operators $\mathfrak{L}_{a}$ annihilate the $S^{1} \times_{q} D^{2}$ partition function for supersymmetric choice of boundary conditions [15]. The partition function takes the form of a multi-residue integral over Wilson line variables $\epsilon_{a}$ after supersymmetric localization $[20,32,33]$. The $Q$-dependent part of 
the integrand represents a vortex sum $\mathcal{I}$ satisfying $\mathfrak{L}_{a} \mathcal{I}=0, a=1, \ldots n_{K}$. For the case of a $3 \mathrm{~d}$ gauge theory with Higgs branch a Kähler manifold $X$, the expansion of $\mathcal{I}$ around the large volume limit $Q_{a}=0$ is a generalized $q$-hypergeometric series

$$
\mathcal{I}=c_{0} \sum_{0 \leq d_{a} \in \mathbb{Z}^{n_{K}}} Q^{\tilde{d}} c(\tilde{d}) q^{C S(d, \epsilon)},
$$

where $\tilde{d}_{a}=d_{a}-\epsilon_{a}, Q^{d}=\prod_{a} Q_{a}^{d_{a}}$ and

$$
c(d)=\frac{\prod_{\beta \in D} \Gamma_{q}\left(1-l_{a \beta} d_{a}\right)}{\prod_{\alpha \in N} \Gamma_{q}\left(1+l_{a \alpha} d_{a}\right)},
$$

in terms of the $q$-Gamma function $\Gamma_{q}(x)$. Moreover, $c_{0}=c(-\epsilon)^{-1}$ and

$$
C S(d, \epsilon)=\frac{1}{2} A_{a b}\left(\left(d_{a}-\epsilon_{a}\right)\left(d_{b}-\epsilon_{b}\right)-\epsilon_{a} \epsilon_{b}\right)+B_{a} d_{a}
$$

is a contribution from the Chern-Simons term in the 3d bulk theory [15].

Example. For the example with the Higgs branch corresponding to the Calabi-Yau threefold $\mathbb{P}_{1,1,2,2,2}^{4}[8]$, we consider the $3 \mathrm{~d}$ GLSM with the gauge group $\mathrm{U}(1)^{2}$ and charged chiral matter fields as summarized by the charge vectors

$$
l_{1}=(-4 ; 1,1,1,1,0,0), \quad l_{2}=(0 ; 0,0,0,-2,1,1) .
$$

Then the difference operators are

$$
\begin{aligned}
& \mathfrak{L}_{1}=\left(1-p_{1}\right)^{3}\left(1-p_{1} p_{2}^{-2}\right)-Q_{1} q^{\frac{1}{2} A_{11}+B_{1}} \prod_{a} p_{a}^{A_{1 a}} \prod_{i=1}^{4}\left(1-p_{1}^{4} q^{i}\right), \\
& \mathfrak{L}_{2}=\left(1-p_{2}\right)^{2}-Q_{2} q^{\frac{1}{2} A_{22}+B_{2}} \prod_{a} p_{a}^{A_{2 a}} \prod_{i=0}^{1}\left(1-p_{1} p_{2}^{-2} q^{-i}\right),
\end{aligned}
$$

with $p_{a}=q^{\theta_{a}}$. The first operator can be factorized as $\mathfrak{L}_{1}=\left(1-p_{1}\right) \mathfrak{L}_{1}^{\prime}$ with

$$
\mathfrak{L}_{1}^{\prime}=\left(1-p_{1}\right)^{2}\left(1-p_{1} p_{2}^{-2}\right)-Q_{1} q^{\frac{1}{2} A_{11}+B_{1}} \prod_{j} p_{j}^{A_{1 j}}\left(\sum_{i=0}^{3}\left(q p_{1}\right)^{i}\right) \prod_{i=1}^{3}\left(1-p_{1}^{4} q^{i}\right) .
$$

$\mathfrak{L}_{1}^{\prime}$ will be the operator that annihilates only the $q$-periods on the hypersurface $X$, as opposed to that on the toric ambient space $W$.

Basis of solutions. Analogous to the Frobenius method for differential equations, the Taylor expansion of $\mathcal{I}$ in the Wilson line parameters $\epsilon_{a}$ generates a set of $N=\operatorname{dim}(K(X))=$ $2\left(1+n_{K}\right)$ linearly independent solutions to the difference operators $\mathfrak{L}_{a}$, where $K(X)$ is the $\mathrm{K}$-group of $X{ }^{2}$ Here linear independence is defined with respect to coefficients invariant

\footnotetext{
${ }^{2}$ By loosely referring to $K(X)$ we really mean the torsion-free K-theory group $K^{0}(X) \otimes \mathbb{Q}$ with coefficients in $\mathbb{Q}$. Using the Chern isomorphism we can then identity the torsion-free K-theory classes with cohomology classes in $H^{2 *}(X, \mathbb{Q})$. Therefore, in the following, the notation $\Phi_{A}$ will denote both the K-theory element and its Chern character, depending on the context.
} 
under the $n_{K}$ shifts $p_{a} Q_{b}=Q_{b} q^{\delta_{a b}}$. In the $2 \mathrm{~d}$ case, a geometric way to construct a vector of solutions is to study the central charges of D-branes. The generalization to E-branes in the 3d GLSM proposed in ref. [15] starts from the geometric interpretation of the residue integral as an integral over $X$ after the replacement

$$
q^{-\epsilon_{a}}=P_{a}=e^{-\beta K_{a}}, \quad q=e^{-\hbar \beta},
$$

where $K_{a} \in H^{2}(X, \mathbb{Z}) \simeq H_{4}(X, \mathbb{Z})$ are the generators of the Kähler cone dual to the Mori cone defined by the charge vectors $l_{a}{ }^{3}$ The parameter $\beta$ is the size of the $S^{1}$ and $\hbar$ is the weight of the twist of the geometry $S^{1} \times{ }_{q} D^{2}[20]$. The $2 \mathrm{~d}$ limit is defined as $\beta \rightarrow 0$. Except for the unusual normalization by an extra factor $\beta$, which comes from the extra circle in the $3 \mathrm{~d}$ theory, $P_{a}$ is the (Chern character of the) line bundle $\mathcal{O}\left(-K_{a}\right)$ on $X$.

The $q$-series $\mathcal{I}$ with values in $K(X)$ obtained from the vortex sum by the replacement (2.2) agrees, for $A_{a b}=0=B_{a}$ and up to an overall factor, with the $I$-function for the permutation symmetric quantum K-theory defined in refs. [4-14]. The case with non-zero $A_{a b}, B_{a}$ is also interesting and includes the more general setup of quantum K-theory at higher level studied in ref. [35].

To obtain a basis of $N$ linearly independent solutions we start with the ring relations among the cohomology elements $K_{a}$ on the toric intersection $X$. These are obtained from the construction of the toric ambient space $W$ as a GIT quotient $W=\left(\mathbb{C}^{\operatorname{dim} W+n_{K}}-\right.$ $\Delta) / /\left(\mathbb{C}^{*}\right)^{n_{K}}$ in a standard way. We refer again to ref. [34] for details. The result is the cohomology ring

$$
H^{2 *}(W, \mathbb{Z})=\mathbb{Z}\left[K_{a}\right] / \Delta_{K},
$$

where $\Delta_{K}$ is the ideal of relations. For simplicity we assume that the cohomology ring $H^{2 *}(X, \mathbb{Q})$ can be generated by products

$$
K_{A}=K_{\vec{a}}=\prod_{i=1}^{k} K_{a_{i}} \in H^{2 *}(W, \mathbb{Z})
$$

of the generators $K_{a}$ of $H^{2 *}(W, \mathbb{Z})$ restricted to the CICY $X$, i.e. that there are no nontoric classes. ${ }^{4}$ Here $A$ runs over the appropriate set of vectors $\vec{a}$ with $\operatorname{dim}(\vec{a}) \leq \operatorname{dim}(X)$ specifying the monomials $K_{A}$ up to degree $\operatorname{dim}(X)$. The restriction of the ideal $\Delta_{K}$ to $X$ gives an ideal $\Delta_{X, K}$ that reduces the set of $\sum_{i=0}^{\operatorname{dim} X}\left(\begin{array}{c}n_{K} \\ i\end{array}\right)$ monomials $K_{\vec{a}}$ to a basis $\left\{K_{A}\right\}$ of dimension $N=\operatorname{dim}(K(X))$.

To this cohomological basis we assign elements $\Phi_{\vec{a}} \in K(X)$ with Chern character ${ }^{5}$

$$
\Phi_{a}=1-e^{-\beta K_{a}}=\beta K_{a}+O\left(\beta^{2}\right), \quad \Phi_{\vec{a}}=\Phi_{a_{1} \ldots a_{k}}=\prod_{i=1}^{k} \Phi_{a_{i}} .
$$

One has $\Phi_{a_{1} \ldots a_{k}} \in H^{\geq 2 k}(X, \mathbb{Q})$. Replacing $K_{a} \rightarrow \Phi_{a}$ in $\left\{K_{A}\right\}$ gives a basis $\left\{\Phi_{A}\right\}$ for $K(X)$. Expanding $\mathcal{I}$ in $\epsilon_{a}$ up to order $\operatorname{dim}(X)$ and reexpressing the result in terms of the basis

\footnotetext{
${ }^{3}$ We refer for instance to the book [34] for background material.

${ }^{4}$ The restricted classes are in general multiples of the generators of $H^{2 *}(X, \mathbb{Z})$ on the CICY $X$; the extra factors become immaterial when passing to coefficients in $\mathbb{Q}$.

${ }^{5}$ The normalization factor $\beta$ from the radius of the extra $S^{1}$ is kept to make the $2 \mathrm{~d}$ limit manifest. To stay within $\mathbb{Q}$-cohomology, $\beta$ should be restricted to rational values.
} 
$\left\{\Phi_{A}\right\}$ gives

$$
\mathcal{I}=\mathcal{I}_{0} \cdot 1+\sum_{a=1}^{n_{K}} \mathcal{I}_{a} \Phi_{a}+\sum_{a \leq b} \mathcal{I}_{a b} \Phi_{a b}+\sum_{a \leq b \leq c} \mathcal{I}_{a b c} \Phi_{a b c}=\sum_{A=0}^{N-1} \mathcal{I}_{A} \Phi_{A}
$$

The coefficients $\mathcal{I}_{A}$ provide a basis of $N$ solutions to the difference equations.

In the $2 \mathrm{~d}$ limit $\beta \rightarrow 0$, the difference operators (2.1) reduce to the GKZ differential operators of $2 \mathrm{~d}$ mirror symmetry, and the $N$ solutions to the difference equations reduce to the ordinary periods associated with the $\mathcal{N}=2$ special geometry of the moduli space. A natural vector of solutions to the difference equations, which reduces to the standard solution vector used in $2 \mathrm{~d}$ mirror symmetry [36] in the small radius limit, ${ }^{6}$ is

$$
\Pi_{q}(Q, q)=\left(\begin{array}{c}
\mathcal{I} \\
-\frac{1}{\hbar} \partial_{K_{a}} \mathcal{I} \\
\frac{1}{2 \hbar^{2}} \kappa_{a b c} \partial_{K_{b}} \partial_{K_{c}} \mathcal{I} \\
-\frac{1}{3 ! \hbar^{3}} \kappa_{a b c} \partial_{K_{a}} \partial_{K_{b}} \partial_{K_{c}} \mathcal{I}
\end{array}\right)=\left(\begin{array}{c}
1 \\
-\frac{1}{\hbar} \ln Q_{a} \\
\frac{1}{2 \hbar^{2}} \kappa_{a b c} \ln Q_{b} \ln Q_{c} \\
-\frac{1}{3 ! \hbar^{3}} \kappa_{a b c} \ln Q_{a} \ln Q_{b} \ln Q_{c}
\end{array}\right) \cdot(1+O(Q)) .
$$

Here $\kappa_{a b c}=\int_{X} K_{a} K_{b} K_{c}$ are the intersection numbers and we used the relations $q=e^{-\beta \hbar}$, $\epsilon_{a}=-K_{a} / \hbar$. The solution vectors in the basis $\left\{K_{A}\right\}$ and the basis $\left\{\Phi_{A}\right\}$ are related by the linear transformation $\mathcal{I}=\sum_{A} \Pi_{q, A} K_{A}=\sum_{A} \mathcal{I}_{A} \Phi_{A}$. The indices on the basis elements $\Phi_{A}$ are raised and lowered with the standard inner product

$$
\left(\Phi_{A}, \Phi_{B}\right)_{K}=\beta^{-\operatorname{dim} X} \int_{X} \Phi_{A} \Phi_{B} \operatorname{td} X=: \chi_{A B} \in \mathbb{Z} .
$$

Example. In the simple example, the ideal $\Delta_{K}=\left\{K_{1}^{3}\left(K_{1}-2 K_{2}\right), K_{2}^{2}\right\}$ also follows from restricting the difference operators $\mathfrak{L}_{a}$ to the degree zero terms of $\mathcal{I}$. On the hypersurface $X \subset W$ one may drop one of the $K_{1}$ factors in the first entry of $\Delta_{K}$. A set of the $N=6$ basis elements for $H^{2 *}(X, \mathbb{Z})$ is then

$$
\left\{K_{A}\right\}:=\left\{1 ; K_{1}, K_{2} ; K_{1}^{2}, K_{1} K_{2} ; K_{1}^{2} K_{2}\right\}, \quad A=0, \ldots 5 .
$$

The intersections are $\kappa_{111}=8, \kappa_{112}=4, \kappa_{122}=\kappa_{222}=0$. The inner product on the basis $\left\{\Phi_{A}\right\}$ induced by the replacement $K_{a} \rightarrow \Phi_{a}$ is ${ }^{7}$

$$
\chi_{A B}=\left(\begin{array}{cccccc}
0 & 6 & 2 & -8 & -2 & 4 \\
6 & -8 & -2 & 8 & 4 & 0 \\
2 & -2 & 0 & 4 & 0 & 0 \\
-8 & 8 & 4 & 0 & 0 & 0 \\
-2 & 4 & 0 & 0 & 0 & 0 \\
4 & 0 & 0 & 0 & 0 & 0
\end{array}\right), \quad \chi^{A B}=\frac{1}{16}\left(\begin{array}{cccccc}
0 & 0 & 0 & 0 & 0 & 4 \\
0 & 0 & 0 & 0 & 4 & 2 \\
0 & 0 & 0 & 4 & -8 & 4 \\
0 & 0 & 4 & 0 & 2 & -1 \\
0 & 4 & -8 & 2 & 0 & 2 \\
4 & 2 & 4 & -1 & 2 & -6
\end{array}\right) \text {. }
$$

K-theoretic mirror map. The $3 \mathrm{~d}$ partition function of the supersymmetric gauge theories is written in the UV variables. To connect this expression to enumerative invariants of ordinary quantum K-theory one needs to determine the flat coordinates in the IR theory.

\footnotetext{
${ }^{6}$ More precisely, the $A$-model is obtained for the special value $\hbar=-2 \pi i$.

${ }^{7}$ The overall factor $\frac{1}{16}$ in $\chi^{-1}$ is related to the comment in fn. 4 .
} 
This step is often called the mirror map in the context of mirror symmetry. The K-theoretic version of the mirror map has been described by Givental in refs. [4-14, 37] as a motion on the Lagrangian cone in the symplectic loop space $\mathcal{K}$ described below. We continue with an outline of the computation in two steps. The first step corresponds to removing the multi-trace deformations in the UV theory, and to shifting the input of the symmetric quantum K-theory to zero. The second step corresponds to a deformation by single trace operators in the gauge theory and determining the flat coordinates for these directions.

As argued in [15], the vortex sum $\mathcal{I}(Q, q)$ of the $3 \mathrm{~d}$ gauge theory takes value in the symmetric quantum K-theory of refs. [4-14]. More precisely it is related to Giventals $I$-function ${ }^{8}$ by the relation

$$
I_{S Q K}(Q, q)=(1-q) \prod_{a} P_{a}^{-\ln Q_{a} / \ln q} \mathcal{I}(Q, q) .
$$

In the symmetric quantum K-theory, the deformations $t_{A}$ are characterized by the expansion

$$
I_{S Q K}(t)=(1-q)+t_{A}(Q, q) \Phi_{A}+\sum_{\vec{d}>0} Q^{\vec{d}}\left\langle\left\langle\frac{\Phi^{A}}{1-q L}\right\rangle\right\rangle_{\vec{d}} \Phi_{A} .
$$

Here $\langle\langle\ldots\rangle\rangle=\sum_{n>0} \frac{1}{n !}\left\langle\ldots, t^{n}\right\rangle$ denotes the correlator part with deformation $t=t_{A} \Phi_{A}$. The split into the input $t$ and the correlator part is defined by the decomposition $\mathcal{K}=\mathcal{K}_{+} \oplus \mathcal{K}_{-}$ where $^{9}$

$$
\begin{aligned}
\mathcal{K} & =K(X) \otimes \mathbb{C}\left(q, q^{-1}\right) \otimes \mathbb{C}[[Q]], \quad \mathcal{K}_{+}=K(X) \otimes \mathbb{C}\left[q, q^{-1}\right] \otimes \mathbb{C}[[Q]], \\
\mathcal{K}_{-} & =K(X) \otimes\{r(q) \in R(q) \mid r(0) \neq \infty \text { and } r(\infty)=0\} \otimes \mathbb{C}[[Q]]
\end{aligned}
$$

such that $t \in \mathcal{K}_{+}$. The input $t(Q, q)$ corresponds to a complicated deformation of the $3 \mathrm{~d}$ theory with multi-traces of Wilson line operators [15]. To obtain correlators in the ordinary quantum K-theory, we first shift this input to zero, by applying Givental's transformation

$$
I(0)=\exp \left(\sum_{r>0} \frac{\Psi_{r}(\epsilon)}{r\left(1-q^{r}\right)}\right) I(t)=(1-q)+0+\sum_{A} F^{A}(0) \Phi_{A},
$$

where $F^{A}(0)$ are the correlator parts at zero deformation. The input $\epsilon=\sum_{A} \epsilon_{A}(Q, q) \Phi_{A}\left(P q^{\theta}\right)$ is determined as a series in $Q$ such that the r.h.s. holds. Here $\epsilon_{a} \in \mathcal{K}_{+}$and $\Phi_{A}\left(P q^{\theta}\right)$ is the operator obtained by replacing $P_{a}$ by $P_{a} q^{\theta_{a}}$ in $\Phi_{A}(P)$. The Adams operator $\Psi_{r}$ acts as

$$
\Psi_{r}(Q)=Q^{r}, \quad \Psi_{r}(q)=q^{r}, \quad \Psi_{r}\left(P_{a}\right)=P_{a}^{r}, \quad \Psi_{r}\left(q^{\theta_{a}}\right)=q^{r \theta_{a}} .
$$

\footnotetext{
${ }^{8}$ One distinguishes the concepts of the Givental $I$-function and the $J$-function, which can be defined using localization methods in the moduli space of quasi-maps and stable maps, respectively. These correspond to the GLSM and the non-linear sigma model, respectively. The two are related by a UV/IR reparameterization, which is part of the $3 \mathrm{~d}$ mirror map discussed below. Despite the fact, that the correlator notation introduced in the following equations refers to the stable map compactification, i.e., the IR phase, we will continue to use the symbol $I$ for the generating function throughout this note also for the IR data.

${ }^{9} \mathcal{K}$ is the symplectic loop space with pairing $\Omega(f, g)=\left(\operatorname{Res}_{q=0}+\operatorname{Res}_{q=\infty}\right) \frac{d q}{q}\left(f(q), g\left(q^{-1}\right)\right)_{K} . \quad R(q)$ denotes the field of rational functions in the variable $q . \mathcal{K}_{ \pm}$are Lagrangian subspaces of $\mathcal{K}$ with respect to the symplectic pairing, see refs. [37, 38].
} 
It is shown in refs. [4-14] that transformations of the form (2.7) generate the deformations on the family of symmetric quantum K-theory parameterized by $t_{A}$ and $Q_{a}$. In the $3 \mathrm{~d}$ gauge theory this transformation arises upon integrating in massive charged degrees of freedom [15]. The series expansion is tedious in practice, but suited to a computation by a symbolic computer program to given order in $Q_{a}$.

In the second step, the $I$-function $I(0)$ can now be deformed again to obtain correlators with deformations of the ordinary, symmetric, or equivariant quantum K-theory [4-14]. The deformation family of ordinary quantum K-theory corresponds to the deformation by single trace operators in the $3 \mathrm{~d}$ field theory [15]. The $I$-function for the ordinary quantum K-theory with input $t_{A}$ is obtained by a transformation of the same type as (2.7) [37], but restricted to the $r=1$ term in the sum:

$$
I_{Q K}(t)=\exp \left(\frac{\epsilon(t)}{1-q}\right) I(0)=(1-q)+\sum_{A} t_{A} \Phi_{A}+\sum_{A} F_{Q K}^{A}(t) \Phi_{A} .
$$

In the following we will restrict to $q$-independent deformations $t_{A} \in \mathbb{C}$, which corresponds to a deformation by operators with (effective) spin zero in the 3 d gauge theory, or to setting the deformations in the direction of gravitational descendants to zero in the quantum K-theory.

Example. Performing the two steps in the example $\mathbb{P}_{1,1,2,2,2}^{4}[8]$ we find the general form (dropping the subscript $Q K$ again)

$$
I\left(t_{A}\right)=(1-q)+t_{A} \Phi_{A}+\sum_{A>n_{K}} F^{A}(t) \Phi_{A} .
$$

The correlators $F^{A}$ are zero in the first $1+n_{K}$ directions $\Phi_{0}=1$ and $\Phi_{a}=\left(1-P_{a}\right)$, as in the cohomological case. Moreover, the correlators depend, except for a classical, $Q$-independent term, only on the deformations $t_{0} \mathbf{1}+\sum_{a=1}^{n_{K}} t_{a} \Phi_{a}$. Since the dependence on $t_{0}$ is universal and determined by the K-theoretic string equation [39], the enumerative invariants will be encoded in the quantum correlators $F^{A}\left(t_{a}\right)$ as functions of the deformations $\sum_{a=1}^{n_{K}} t_{a} \Phi_{a}$.

It is useful to express the correlators in terms of the dual basis

$$
F^{A} \Phi_{A}=\left(F_{A, c l}+\hat{F}_{A}\right) \Phi^{A}, \quad \hat{F}_{A}=\sum_{\vec{d}>0} Q^{\vec{d}}\left\langle\left\langle\frac{\Phi_{A}}{1-q L}\right\rangle\right\rangle_{\vec{d}},
$$

where $F_{A, c l}$ denotes the classical contribution ${ }^{10}$ and $\hat{F}_{A}$ the quantum correlators. The precise form of the correlators depends on the choice of Chern-Simons terms. For zero effective levels $A_{a b}=0=B_{a}$ we find for the 1-point functions, to leading order in the degrees $Q_{a}$ :

$$
\begin{aligned}
& \hat{F}_{0}=-\frac{640(3 q-1) Q_{1}}{(q-1)^{2}}+\frac{4(1-3 q) Q_{2}}{(q-1)^{2}}-\frac{16\left(1761 q^{4}+5016 q^{3}+4122 q^{2}-787\right) Q_{1}^{2}}{(q-1)^{2}(q+1)^{3}}+\ldots, \\
& \hat{F}_{1}=\frac{640 Q_{1}}{1-q}+\frac{32\left(607 q^{2}+1254 q+667\right) Q_{1}^{2}}{(1-q)(q+1)^{2}}+\frac{640 Q_{1} Q_{2}}{1-q}+\ldots, \\
& \hat{F}_{2}=\frac{4 Q_{2}}{1-q}+\frac{640 Q_{1} Q_{2}}{1-q}-\frac{4\left(q^{2}-2\right) Q_{2}^{2}}{(1-q)(q+1)^{2}}+\ldots
\end{aligned}
$$

\footnotetext{
${ }^{10}$ See eq. (2.19) below.
} 
We will not give more explicit results at this point, since we found the universal formula eq. (2.10) below for the quantum correlators of $\mathbb{P}_{1,1,2,2,2}^{4}[8]$, which holds also for all other Calabi-Yau threefolds that we studied so far (and with modifications also for Calabi-Yau manifolds of other dimensions, see section 3).

\subsection{Multi-cover formula for Calabi-Yau threefolds}

Below we propose a formula that gives the quantum correlators of the Calabi-Yau threefold $X$ in ordinary quantum $\mathrm{K}$-theory at level zero in terms of the Gopakumar-Vafa invariants for $X$, or vice versa. In the next section we derive the formula for the resolved conifold, which has only a single isolated curve of degree one. The general formula extrapolates the K-theoretic multi-cover formula of the conifold to higher degree maps, similarly to what has been done in the $2 \mathrm{~d}$ context in ref. [40].

We have explicitly checked the proposed multi-covering formula up to a certain degree in $Q$ for Calabi-Yau threefolds $X_{3}$ with up to three Kähler moduli, including the examples

$$
\begin{array}{ll}
h^{1,1}=1: & \mathbb{P}_{1,1,1,1,1}^{4}[5], \mathbb{P}_{1,1,1,1,2}^{4}[6], \mathbb{P}_{1,1,1,1,1,1}^{5}[4,2], \\
h^{1,1}=2: & \mathbb{P}_{1,1,2,2,2}^{4}[8], \mathbb{P}_{1,1,2,2,6}^{4}[12], \mathbb{P}_{1,1,1}^{2}[3] \rightarrow X_{3} \rightarrow \mathbb{P}^{2} \\
h^{1,1}=3: & \mathbb{P}_{1,2,3}^{2}[6] \rightarrow X_{3} \rightarrow \mathbb{F}_{k}, k=1,2 .
\end{array}
$$

The last three examples are elliptic fibrations over the base $B=\mathbb{P}^{2}$ and the Hirzebruch surfaces $B=\mathbb{F}_{k}$, respectively. In these cases we checked, that in the limit of large elliptic fiber one obtains the invariants for the local Calabi-Yau threefolds given by the cotangent bundles of the base $B$, e.g., $\mathcal{O}(-3)_{\mathbb{P}^{2}}$ in the first case. The above examples, including the main example $\mathbb{P}_{1,1,2,2,2}^{4}[8]$, do not only contain isolated curves but also families. The numerical verifications give evidence for the proposed formula, but we do not have a mathematical proof. From the physics point of view, formula (2.10) says that the counting of BPS objects on the $3 \mathrm{~d}$ world-volume and of $5 \mathrm{~d}$ BPS objects in the target space differ only in the contribution of multi-covers in $n<3$-pt functions. It would be interesting to derive this difference from a membrane/target space duality.

Conjecture. The genus zero correlators at non-zero degree $\vec{d}>0$ of ordinary quantum K-theory (at level zero) on Calabi-Yau threefold $X$ are related to the Gopakumar-Vafa invariants $\mathfrak{n}_{\vec{d}}$ for $X$ as

$$
\begin{aligned}
& \hat{F}_{0}=p_{2}+\frac{1}{(1-q)^{2}}\left[(1-3 q) \mathcal{F}+q \sum_{a} t_{a} \mathcal{F}_{a}\right]_{t^{n>2}}, \\
& \hat{F}_{a}=p_{1, a}+\frac{1}{(1-q)}\left[\mathcal{F}_{a}\right]_{t^{n>1}}, \quad a=1, \ldots n_{K}, \\
& \hat{F}_{A}=0, \quad A>n_{K} .
\end{aligned}
$$

Here

$$
\mathcal{F}\left(Q_{a} e^{t_{a}}\right)=\sum_{\vec{d}>\overrightarrow{0}, n \geq 0} Q^{\vec{d}} \frac{\left(\sum_{j} d_{j} t_{j}\right)^{n}}{n !} \sum_{r \mid \vec{d}} \frac{\mathfrak{n}_{\vec{d} / r}}{r^{3}}, \quad \mathcal{F}_{a}=\partial_{t_{a}} \mathcal{F},
$$


is the potential for the Gromov-Witten invariants, which depends only on the combinations $Q_{a} e^{t_{a}}$, and $[f]_{t^{n>n^{\prime}}}$ denotes $f$ with the terms of degree $\leq n^{\prime}$ in $t_{a}$ dropped. Moreover

$$
\begin{gathered}
p_{1, a}=\sum_{\vec{d}>\overrightarrow{0}} Q^{\vec{d}} \sum_{r \mid \vec{d}} \mathfrak{n}_{\vec{d} / r} \cdot\left\{\frac{d_{a}}{r}(1-q) \frac{r\left(1-q^{r}\right)+q^{r}}{\left(1-q^{r}\right)^{2}}+\frac{d_{a}}{r^{2}}\left(\sum_{j} t_{j} d_{j}\right) \frac{1}{\left(1-q^{r}\right)}\right\}, \\
p_{2}=\sum_{\vec{d}>\overrightarrow{0}} Q^{\vec{d}} \sum_{r \mid \vec{d}} \mathfrak{n}_{\vec{d} / r} \cdot\left\{(1-q) \frac{r^{2}\left(1-q^{r}\right)^{2}-q^{r}\left(1+q^{r}\right)}{\left(1-q^{r}\right)^{3}}\right. \\
\left.+\frac{\sum_{j} d_{j} t_{j}}{r} \frac{r\left(1-q^{r}\right)-q^{r}}{\left(1-q^{r}\right)^{2}}+\frac{\left(\sum_{j} d_{j} t_{j}\right)^{2}}{2 r^{2}(1-q)}\right\} .
\end{gathered}
$$

The K-theoretic $n$-point functions with $n \geq 3$ are directly related to the Gromov-Witten prepotential $\mathcal{F}$ for $X$, as in the one modulus case considered in ref. [15]. The coefficients of these $n$-point functions are, up to an overall power of $(1-q)$, the same as that of the cohomological expansion and integral.

On the contrary, the expansion of the $n<3$ point functions have non-integral coefficients in the cohomological theory, while they are integral in quantum K-theory. From the perspective of the $3 \mathrm{~d}$ gauge theory, the integrality arises from the interpretation of the supersymmetric partition function as a BPS index on the $3 \mathrm{~d}$ world-volume. The integral $3 \mathrm{~d}$ BPS invariants are encoded in the polynomials $p_{1, a}$ and $p_{2}$ of degree 1 and 2 in the deformations $t_{a}$, respectively.

Eqs. (2.10), (2.12) apply for the canonical choice $A=0=B$ for the Chern-Simons terms in the $3 \mathrm{~d}$ theory. For other Chern-Simons terms one obtains a similar relation between the two types of invariants, but the $q$-dependence of the multi-cover contributions from higher degree maps is no longer the same as for degree one maps. The classical terms in the $q$-periods are given in eq. (2.19). It is straightforward to verify, that the $3 \mathrm{~d}$ expressions reduce to the $2 \mathrm{~d}$ period vector of $X$ in flat coordinates in the small radius limit.

\section{$2.3 \quad$ Local conifold}

The simplest Calabi-Yau threefold with non-trivial quantum corrections is the local conifold described by the GLSM with charge vector $q_{a}=(1,1,-1,-1)$ and no hypersurface constraint. This is the non-compact threefold $X$ that contains a single rational curve of degree one with normal bundle $\mathcal{O}(-1) \oplus \mathcal{O}(-1)$. In order to extract correlators from the $I$-function we need to regularize the non-compact directions. This can be achieved by either introducing a real mass $m$ for the negatively charged fields of the GLSM, or by embedding the local geometry into a global one. We first discuss the mass deformation. The real mass $m$ becomes the weight with respect to the $\mathrm{U}(1)$ flavor symmetry rotating the two chiral fields parametrizing the normal direction $\mathcal{O}(-1) \oplus \mathcal{O}(-1)$. On the level of geometry the $\mathrm{U}(1)$ flavor symmetry becomes an $S^{1}$-action on the non-compact threefold $X$, which multiplies the fibers of the normal bundle $\mathcal{O}(-1) \oplus \mathcal{O}(-1)$ with a phase, and the fugacity $y=e^{m}$ of the flavor symmetry realizes the equivariant parameter of the equivariant K-group $K_{S^{1}}(X)$, which is given by

$$
K_{S^{1}}(X) \simeq K\left(\mathbb{P}^{1}\right)\left[y, y^{-1}\right] \simeq \mathbb{Q}\left[P, y, y^{-1}\right] /(1-P)^{2}
$$


The strategy is now to compute equivariant correlator functions and then take the limit $y \rightarrow 1$. For the given geometry and in terms of the K-theoretic equivariant Euler class $e_{S^{1}}^{K}=\left(1-y P^{-1}\right)^{2}$ of the normal bundle $\mathcal{O}(-1) \oplus \mathcal{O}(-1)$ to $\mathbb{P}^{1}$ the equivariant holomorphic Euler characteristic becomes ${ }^{11}$

$$
\chi_{S^{1}}(X, E)=\chi\left(\mathbb{P}^{1}, \frac{\left.E\right|_{\mathbb{P}^{1}}}{e_{S^{1}}^{K}}\right) \in \mathbb{Q}\left[y, y^{-1}\right] .
$$

Taking the limit $y \rightarrow 1$, which amounts to setting the real mass $m$ to zero, allows us to extract regularized holomorphic Euler characteristics of the non-compact threefold $X$. As a consequence the inner product is defined as

$$
\left(\Phi_{A}, \Phi_{B}\right)_{K}=\lim _{y \rightarrow 1} \chi_{S^{1}}\left(X, e_{S^{1}}^{K} \Phi_{A} \Phi_{B}\right)=\chi\left(\mathbb{P}^{1}, \Phi_{A} \Phi_{B}\right)=\delta_{A+B, 0}+\delta_{A+B, 1},
$$

with $\Phi_{0}=1$ and $\Phi_{1}=1-P$.

Then the equivariant $I$-function of the symmetric theory for the conifold reads [4-14]

$$
\begin{aligned}
I_{S Q K} & =(1-q)\left(1+\sum_{d=1}^{+\infty} Q^{d} \frac{\prod_{n=0}^{d-1}\left(1-y P^{-1} q^{-n}\right)^{2}}{\prod_{n=1}^{d}\left(1-P q^{n}\right)^{2}}\right) \\
& =(1-q)\left[1+e_{S^{1}}^{K}\left(\sum_{d=1}^{+\infty} \frac{Q^{d}}{q^{d(d-1)} P^{2 d-2}\left(1-P q^{d}\right)^{2}}+O(1-y)\right)\right] \\
& =(1-q)\left[1+e_{S^{1}}^{K}\left(I_{0} \Phi_{0}+I_{1} \Phi_{1}+O(1-y)\right)\right]
\end{aligned}
$$

where in the second and third line we have not displayed the terms of order $O(1-y)$, as these terms eventually vanish in the limit $y \rightarrow 1$, and with

$$
I_{0}=\sum_{d=1}^{+\infty} \frac{Q^{d}}{\left(1-q^{d}\right)^{2} q^{d(d-1)}}, \quad I_{1}=\sum_{d=1}^{+\infty} \frac{2 Q^{d}}{\left(1-q^{d}\right)^{2} q^{d(d-1)}}\left(d-\frac{1}{1-q^{d}}\right) .
$$

Extracting the $\mathcal{K}_{+}$part of these expressions we find for the input $t_{0} \Phi_{0}+t_{1} \Phi_{1}$ of the symmetric quantum K-theory the expressions

$$
t_{0}=(1-q) e_{S^{1}}^{K} \sum_{n=1}^{d-1} n q^{d(n-d)}, \quad t_{1}=(1-q) e_{S^{1}}^{K} \sum_{n=1}^{d-1} n(2 d-n-1) q^{d(n-d)},
$$

such that the $I$-function becomes

$$
\begin{aligned}
I_{S Q K}=(1-q)+\left(t_{0} \Phi_{0}+\right. & \left.t_{1} \Phi_{1}\right) \\
+(1-q) e_{S^{1}}^{K} \sum_{d=1}^{+\infty} Q^{d} & {\left[\Phi_{0} \frac{d\left(1-q^{d}\right)+q^{d}}{\left(1-q^{d}\right)^{2}}\right.} \\
& \left.+\Phi_{1} \frac{d(d-1)\left(1-q^{d}\right)^{2}-2 q^{d}}{\left(1-q^{d}\right)^{3}}+O(1-y)\right],
\end{aligned}
$$

where the terms in the square bracket reside in the $\mathcal{K}_{-}$part.

\footnotetext{
${ }^{11}$ For ease of notation we set $\beta=1$ in this subsection.
} 
We observe that the input $t_{0} \Phi_{0}+t_{1} \Phi_{1}$ is proportional to equivariant K-theoretic Euler class $e_{S^{1}}^{K} \sim O(1-y)$. Hence, the input of the $I$-function vanishes in the limits $y \rightarrow$ 1 , and therefore only contributes to the equivariant terms $O(1-y)$ in $\mathcal{K}_{-}$in eq. (2.15) (which we have not spelled out explicitly). Upon removing this input with a suitable transformation (2.7), we therefore arrive at the $I$-function

$$
\begin{aligned}
I=(1-q)+(1-q) e_{S^{1}}^{K} \sum_{d=1}^{+\infty} Q^{d}\left[\begin{array}{l}
\Phi_{0} \\
\end{array}\right. & \frac{d\left(1-q^{d}\right)+q^{d}}{\left(1-q^{d}\right)^{2}} \\
& \left.+\Phi_{1} \frac{d(d-1)\left(1-q^{d}\right)^{2}-2 q^{d}}{\left(1-q^{d}\right)^{3}}+O(1-y)\right] .
\end{aligned}
$$

From this expression together with the metric (2.13) we readily read off in the limit $y \rightarrow 1$ the 1-pt functions ${ }^{12}$

$$
\begin{aligned}
\left\langle\frac{\Phi_{1}}{1-q L}\right\rangle_{d} & =(1-q) \frac{d\left(1-q^{d}\right)+q^{d}}{\left(1-q^{d}\right)^{2}}, \\
\left\langle\frac{\Phi_{0}}{1-q L}\right\rangle_{d} & =(1-q) \frac{d^{2}\left(1-q^{d}\right)^{2}-q^{d}\left(1+q^{d}\right)}{\left(1-q^{d}\right)^{3}} .
\end{aligned}
$$

We can now generate the non-trivial input $t \Phi_{1}$ for the ordinary quantum K-theory by acting with the transformation $\exp \left(\frac{t\left(1-P q^{\theta}\right)}{1-q}\right)$ according to ref. [37], which yields the $I$-function

$$
I_{Q K}(t)=(1-q)+K_{0}(Q, q, t) \Phi_{0}+K_{1}(Q, q, t) \Phi_{1}+e_{S^{1}}^{K} O(1-y),
$$

where $K_{\ell}(Q, q, t), \ell=1,2$, decompose into the input $K_{\ell}^{+}(Q, q, t)$ and the correlator contributions $K_{\ell}^{-}(Q, q, t)$. A straightforward but tedious computation yields the (for us relevant) input

$$
K_{0}^{+}(Q, q, t)=e_{S^{1}}^{K} \sum_{d=2}^{\infty} \sum_{n=1}^{+\infty} \frac{t^{n} Q^{d}}{n !} k_{d, n}^{+}(q), \quad K_{1}^{+}(Q, q, t)=t+e_{S^{1}}^{K}(\ldots),
$$

with

$$
k_{d, n}^{+}(q)= \begin{cases}(d-1)+O(1-q) & \text { for } d \geq 2, n=1 \\ \frac{d^{n-2}(d-1)(2 d-n+2)}{2}+O(1-q) & \text { for } d \geq 2, n>1 \\ 0 & \text { else }\end{cases}
$$

Furthermore, the correlator contributions read

$$
\begin{aligned}
K_{0}^{-}(Q, q, t)= & e_{S^{1}}^{K} \sum_{d=0}^{+\infty} Q^{d}\left[(1-q) \frac{d\left(1-q^{d}\right)+q^{d}}{\left(1-q^{d}\right)^{2}}+\frac{t}{1-q^{d}}+\sum_{n=2}^{+\infty} \frac{d^{n-2} t^{n}}{n !(1-q)}\right], \\
K_{1}^{-}(Q, q, t)= & e_{S^{1}}^{K} \sum_{d=0}^{+\infty} Q^{d}\left[(1-q) \frac{d(d-1)\left(1-q^{d}\right)^{2}-2 q^{d}}{\left(1-q^{d}\right)^{3}}+\frac{t\left(d\left(1-q^{d}\right)-1\right)}{\left(1-q^{d}\right)^{2}}+\frac{t^{2}(d-1)}{1-q}\right. \\
& \left.+\sum_{n=3}^{+\infty} \frac{t^{n}}{n !}\left(\frac{d^{n-3}(n-2)}{(1-q)^{2}}+\frac{d^{n-3}(n-3)(n-2)}{2(1-q)}-\frac{d^{n-2}\left(2-n-2 d n+n^{2}\right)}{2(1-q)}\right)\right] .
\end{aligned}
$$

\footnotetext{
${ }^{12}$ Note that, in principal, the permutation symmetric input $t_{0} \Phi_{0}+t_{1} \Phi_{1}$ yields higher point degree zero correlators, which - due to the $Q$-dependence of this input — potentially contributes to $\mathcal{K}_{-}$at the same order in $Q$ as the extracted 1-pt correlators. However, in the limit $y \rightarrow 0$ all these contributions vanish.
} 
Due to the $Q$-dependent terms in the input in $K_{0}^{+}$, we see that the $n$-pt function in $K_{0}^{-}$at degree $d$ combines with 2-pt functions at degree 0 , such that we obtain with the metric (2.13) the equations

$$
\begin{aligned}
\sum_{n=0}^{+\infty} \frac{t^{n} Q^{d}}{n !}\left\langle\frac{\Phi_{1}}{1-q L}, \Phi_{1}^{n}\right\rangle_{d} & =\left.K_{0}^{-}(Q, q, t)\right|_{Q^{d}}, \\
\sum_{n=0}^{+\infty} \frac{t^{n} Q^{d}}{n !}\left\langle\frac{\Phi_{0}}{1-q L}, \Phi_{1}^{n}\right\rangle_{d} & =\left.K_{0}^{-}(Q, q, t)\right|_{Q^{d}}+\left.K_{1}^{-}(Q, q, t)\right|_{Q^{d}} \\
& -\sum_{n=2}^{+\infty} \frac{t^{n} Q^{d}}{(n-1) !} k_{d, n-1}^{+}(1)\left\langle\frac{\Phi_{0}}{1-q L}, e_{S^{1}}^{K} \Phi_{0}, \Phi_{1}\right\rangle_{0} .
\end{aligned}
$$

The appearing degree zero correlator reduces to the ordinary quantum K-theory correlator of the point, such that we get with eq. $(2.13)^{13}$

$$
\left\langle\frac{\Phi_{0}}{1-q L}, e_{S^{1}}^{K} \Phi_{0}, \Phi_{1}\right\rangle_{0}=\chi_{S^{1}}\left(X, e_{S^{1}}^{K} \Phi_{1}\right)\left\langle\frac{1}{1-q L}, 1,1\right\rangle_{\mathrm{pt}}=\frac{1}{1-q} .
$$

These two identities allow us to derive the $(n+1)$-pt functions of the ordinary quantum K-theory, which turn out to be

$$
\begin{array}{lll}
\left\langle\frac{\Phi_{1}}{1-q L}, \Phi_{1}\right\rangle_{d}=\frac{1}{1-q^{d}}, & \left\langle\frac{\Phi_{1}}{1-q L}, \Phi_{1}^{n}\right\rangle_{d}=\frac{d^{n-2}}{1-q} & \text { for } n>1, \\
\left\langle\frac{\Phi_{0}}{1-q L}, \Phi_{1}\right\rangle_{d}=\frac{d\left(1-q^{d}\right)-q^{d}}{\left(1-q^{d}\right)^{2}}, & \left\langle\frac{\Phi_{0}}{1-q L}, \Phi_{1}^{2}\right\rangle_{d}=\frac{1}{1-q}, & \\
\left\langle\frac{\Phi_{0}}{1-q L}, \Phi_{1}^{n}\right\rangle_{d}=d^{n-3} \frac{1-q(3-n)}{(1-q)^{2}} & \text { for } n>2 .
\end{array}
$$

The correlators in eqs. (2.16) and (2.17) sum up to the expression (2.10) for $n_{K}=1$ and $\mathfrak{n}_{k>0}=0$.

A geometric regularization of the non-compact directions, which is in the spirit of local mirror symmetry, is to embed the local conifold geometry into a global Calabi-Yau threefold and study its decompactification limit. A simple compactification of the local conifold is the elliptic fibration $X$ over the Hirzebruch surface $\mathbb{F}_{1}$ described by a $3 \mathrm{~d}$ GLSM with gauge group $\mathrm{U}(1)^{3}$ and matter fields with charge vectors

$$
l_{1}=(-6 ; 3,2,1,0,0,0,0), l_{2}=(0 ; 0,0,-2,1,1,0,0), l_{3}=(0 ; 0,0,-1,0,-1,1,1) .
$$

In the limit of large Kähler classes $Q_{1,2} \rightarrow 0$, the elliptic fiber and the $\mathbb{P}^{1}$ fiber of $\mathbb{F}_{1}$ decompactify and the local geometry of the compact $\mathbb{P}^{1}$ is the one studied above. Consider the $q$-hypergeometric series $\mathcal{I}$ which solves the system of difference equations eq. (2.1) for the compact manifold $X$. The leading term in the decompactification limit is $\mathcal{I}_{\text {lim }}=$ $\lim _{Q_{1,2} \rightarrow 0} \mathcal{I}=Q_{1}^{\epsilon_{1}} Q_{2}^{\epsilon_{2}} I_{\text {local }}\left(Q_{3}\right)$. The only non-trivial difference equation in this limit arises

\footnotetext{
${ }^{13}$ As a result of the degree zero correlator only $k_{d, n-1}^{+}(q)$ at $q=1$ contributes to $\mathcal{K}^{-}$. Furthermore, we note that the correlator $\left\langle\frac{\Phi_{0}}{1-q L}, e_{S^{1}}^{K} \Phi_{1}, \Phi_{1}\right\rangle_{0}$ vanishes.
} 
from the difference operator $\mathfrak{L}_{3}=\left(1-p_{3}\right)^{2}-Q_{3}\left(1-p_{3}^{-1} p_{2}\right)\left(1-p_{3}^{-1} p_{1} p_{2}^{-2}\right)$. Applying $\mathfrak{L}_{3}$ to $\mathcal{I}_{\text {lim }}$ and dividing by $Q_{1}^{\epsilon_{1}} Q_{2}^{\epsilon_{2}}$ we obtain the difference equation

$$
\mathfrak{L}_{\text {lim }} I_{\text {local }}(Q)=0, \quad \mathfrak{L}_{\lim }=(1-p)^{2}-Q\left(1-p^{-1} y_{1}\right)\left(1-p^{-1} y_{2}\right) .
$$

Here we used $Q=Q_{3}, p=p_{3}, y_{1}=q^{\epsilon_{2}}$ and $y_{2}=q^{\epsilon_{1}-2 \epsilon_{2}}$. The difference operator $\mathfrak{L}_{\lim }$ is the same as the difference operator for the equivariant theory of the local conifold for real masses $y_{1}, y_{2}$ of the non-compact directions. In particular, specializing to $y=y_{1}=y_{2}$, $\mathfrak{L}_{\lim }$ annihilates the equivariant $I$-function $(2.14) .{ }^{14}$ This shows the equivalence of the two regularizations.

One can also compute directly in the non-equivariant limit. Noticing that the nonequivariant limit of the relation $e_{S^{1}}^{K}(1-P)^{2}=0$ is $(1-P)^{4}=0$, one arrives at a degree four difference operator for the non-equivariant conifold of the form

$$
\mathfrak{L}_{\mathrm{lcf}}=(1-p)^{2}\left(1-z p^{-2}\right)(1-p)^{2}
$$

The solutions of $\mathfrak{L}_{\mathrm{lcf}}$ are the coefficients of the $q$-hypergeometric series $\mathcal{I}$ in eq. (2.14) at $y=1$ in an expansion up to order $(1-P)^{3}$. From here, the computation in the non-equivariant theory proceeds as in the previous examples and leads to the same result eqs. (2.16), (2.17).

The conifold example captures the contribution for a rigid rational curve of degree one with normal bundle $\mathcal{O}(-1) \oplus \mathcal{O}(-1)$. The general formula eq. (2.10) predicts, that the K-theoretic multi-cover contributions from other curves are of the same universal form, up to extra combinatorial factors of the degree $d_{a}$ (which have to be consistent with the 2 dimit). E.g. the $\mathbb{P}_{1,1,2,2,2}^{4}[8]$ contains a family of rational curves of degree $\vec{d}=(0,1)$ with normal bundle $\mathcal{O}(-2) \oplus \mathcal{O}(0)$. The local model for this curve can be obtained as the non-compact limit $Q_{1} \rightarrow 0$ of the compact threefold, and it gives the same multi-cover formula as for the rigid curve.

\subsection{K-theoretic ring}

The quantum correlators (2.10) determine the quantum deformation of the multiplication rings in quantum $\mathrm{K}$-theory. Adding the classical terms to the quantum correlators, the $q$-period vector in the basis $\left\{\Phi_{0}, \Phi_{a}, \Phi^{a}, \Phi^{0}\right\}$ is

$$
\Pi(Q, t)=\left(\begin{array}{c}
\mathfrak{q} \\
\hat{t}_{a} \\
F_{a, c l}+p_{1, a}+\frac{1}{\mathfrak{q}}\left[\mathcal{F}_{a}\right]_{t^{n>1}} \\
F_{0, c l}+p_{2}+\frac{1}{\mathfrak{q}^{2}}\left[(1-3 q) \mathcal{F}+q \sum_{a} t_{a} \mathcal{F}_{a}\right]_{t^{n>2}}
\end{array}\right)
$$

\footnotetext{
${ }^{14}$ To compensate for the fact that eq. (2.14) is written without the overall factor $P^{-\ln Q / \ln q}, p$ has to be taken to be $p=P q^{\theta}$.
} 
Here $\mathfrak{q}=1-q$ and

$$
\begin{aligned}
F_{a, c l}= & \kappa_{a b c}\left(\frac{1}{2 \mathfrak{q}} \hat{t}_{b} \hat{t}_{c}-\mathfrak{q} \frac{L_{b}}{2} \delta_{b c}\right)+\sum_{A>n_{K}} t_{A} \chi_{A a}, \\
F_{0, c l}= & \kappa_{a b c}\left(\frac{1}{3 ! \mathfrak{q}^{2}} \hat{t}_{a} \hat{t}_{b} \hat{t}_{c}-\frac{\delta_{a c}}{2} L_{a} \hat{t}_{b}-\mathfrak{q} \frac{\delta_{a b} \delta_{a c}}{3} L_{a}\right) \\
& +\frac{\chi_{A B}}{2 \mathfrak{q}} \hat{t}_{A} \hat{t}_{B}-\mathfrak{q} \frac{L_{a}}{2} \chi_{a a}+\sum_{A>n_{K}} t_{A} \chi_{A 0},
\end{aligned}
$$

where the small and capital indices run over the sets $a=1, \ldots, n_{K}$ and $A=0, \ldots$, $\operatorname{dim}(K(X))-1$, respectively, and the shifted variables $\hat{t}_{A}$ are given by

$$
\hat{t}_{A}=t_{A}-\delta_{A a} \mathfrak{q} L_{a}, \quad L_{a}=\frac{\ln Q_{a}}{\ln q} .
$$

The period matrix $\pi$ defined from $\Pi$ is

$$
\pi=\left(\begin{array}{c}
\pi_{0} \\
\pi_{1, a} \\
\pi_{2, \mu} \\
\pi_{*}
\end{array}\right)=\left(\begin{array}{r}
\Pi^{T} \\
\mathfrak{q} \partial_{t_{a}} \Pi^{T} \\
\mathfrak{q} \partial_{t_{\mu}} \Pi^{T} \\
\mathfrak{q} \partial_{t_{*}} \Pi^{T}
\end{array}\right)
$$

for the deformation $t=\sum_{A>0} t_{A} \Phi_{A}=t_{a} \Phi_{a}+t_{\mu} \Phi_{n_{K}+\mu}+t_{*} \Phi_{1+2 n_{K}}$. Here we use $t_{a}$, $a=1, \ldots n_{K}, t_{\mu}, \mu=1, \ldots n_{K}$ and $t_{*}$ to denote the deformations in the directions of the basis elements with minimal cohomological degree two, four and six, respectively.

The flatness of the connection in the $t$-directions can be expressed in terms of the linear differential equations for the period matrix

$$
\mathfrak{q} \partial_{t_{A}} \pi(Q, t)=C_{A}(Q, t) \pi(Q, t) .
$$

Here $C_{A}(Q, t)$ are the matrices of structure constants. Starting from (2.18) one finds

$$
\mathfrak{q} \partial_{t_{a}}\left(\begin{array}{c}
\pi_{0} \\
\pi_{1, b} \\
\pi_{2, \mu} \\
\pi_{*}
\end{array}\right)=\left(\begin{array}{cccc}
0 & \delta_{a c} & 0 & 0 \\
0 & 0 & \hat{C}_{a b}^{\nu} & \hat{c}_{a b} \\
0 & 0 & 0 & \hat{\chi}_{a \mu} \chi_{0 *}^{-1} \\
0 & 0 & 0 & 0
\end{array}\right)\left(\begin{array}{c}
\pi_{0} \\
\pi_{1, c} \\
\pi_{2, \nu} \\
\pi_{*}
\end{array}\right)
$$

and

$$
\mathfrak{q} \partial_{t_{\mu}} \pi=\left(\begin{array}{cccc}
0 & 0 & \delta_{\mu \nu} & 0 \\
0 & 0 & 0 & \hat{\chi}_{b \mu} \chi_{0 *}^{-1} \\
0 & 0 & 0 & 0 \\
0 & 0 & 0 & 0
\end{array}\right) \pi, \quad \mathfrak{q} \partial_{t_{*}} \pi=\left(\begin{array}{cccc}
0 & 0 & 0 & 1 \\
0 & 0 & 0 & 0 \\
0 & 0 & 0 & 0 \\
0 & 0 & 0 & 0
\end{array}\right) \pi
$$

where

$$
\begin{aligned}
\hat{C}_{a b}^{\nu} \hat{\chi}_{\nu c} & =\kappa_{a b c}+\mathcal{F}_{a b c}=: C_{a b c}^{G W}\left(Q e^{t}\right), \\
\hat{c}_{a b} \chi_{0 *} & =\left[\mathcal{F}_{a b}\right]_{t^{n>0}}-\mathcal{F}_{a b c} t_{c}+\chi_{a b}-\hat{C}_{a b}^{\nu} \chi_{\nu 0}+\mathfrak{q} p_{2, a b} .
\end{aligned}
$$


Here $C_{a b c}^{G W}$ are the structure constant of the GW theory, which depend only on the combinations $Q_{a} e^{t_{a}}$ of the parameters $(Q, t)$. The $n_{K} \times n_{K}$ matrix $(\hat{\chi})_{c \nu}:=\left(\Phi_{c}, \Phi_{\nu+n_{k}}\right)_{K}$ is invertible and $\chi_{0 *}=\left(\Phi_{0}, \Phi_{1+2 n_{k}}\right)_{K}$ is non-zero, i.e. the above relations can be solved for $\hat{C}$ and $\hat{c}$.

From the above it follows, that the only $3 \mathrm{~d}$ product with non-trivial quantum deformation is

$$
\Phi_{a} \otimes \Phi_{b}=\hat{C}_{a b}^{\nu} \Phi_{\nu}+\hat{c}_{a b} \Phi_{*},
$$

where $\hat{C}_{a b}^{\nu}=\hat{\chi}^{\nu c} C_{a b c}^{G W}$ are determined by the structure constants of the cohomological theory, and the second term $\hat{c}_{a b}$ depends in addition on the $n \leq 2$-point correlators expressed in terms of the cohomological invariants in eq. (2.10).

The matrices $C_{A}$ satisfy the flatness relations $\left[C_{A}, C_{B}\right]=0=\partial_{t_{A}} C_{B}-\partial_{t_{B}} C_{A}$, which follow from the WDVV equations of quantum K-theory [41, 42], or, from the point of the underlying $3 \mathrm{~d}$ field theory, the $3 \mathrm{~d} t t^{*}$ equations [21]. An iteration of the first order system (2.4) yields a differential equation for $\pi_{0}(Q, t)$ in the $t$-parameters, which is the $3 \mathrm{~d}$ analogue of the Picard-Fuchs equation in flat coordinates. The period matrix also satisfies a first order difference system in the $Q$-parameters. It iterates to a system of difference equations for $\pi_{0}(Q, t)$, which is a deformation of eq. (2.1) by the $t$-parameters. Together, these difference and differential equations determine the dependence of $\pi_{0}(Q, t)$ on the two types of parameters $Q$ and $t$. For more details on this deformation space in the context of the $3 \mathrm{~d}$ gauge theory and a discussion of the multiplication ring associated with the difference operators in $Q$ we refer to ref. [15].

\section{Other dimensions}

Dimensions other than three are also interesting for several reasons. For dimension less than three, i.e. for $T^{2}$ and K3 manifolds, we find that the $I$ function in ordinary quantum $\mathrm{K}$-theory computed as above is the classical one

$$
I\left(t_{A}\right)=(1-q)+t_{A} \Phi_{A}+\sum_{A>n_{K}} F_{c l}^{A}(t) \Phi_{A}
$$

where the last term is zero for $T^{2}$. There is still interesting non-perturbative information in the symmetrized, or more generally permutation equivariant, theory. In particular, for all dimensions, the $3 \mathrm{~d}$ vortex $\operatorname{sum} \mathcal{I}(Q, q)$ is non-trivial and computes the coefficients of the ordinary $2 \mathrm{~d}$ mirror map in terms of the integral degeneracies of $3 \mathrm{~d}$ BPS states, as discussed in section 8.3 of ref. [15].

For dimension higher than three, the cohomological Gromov-Witten invariants can be still be computed from the entries $\Pi_{\gamma}^{\ln ^{2}}\left(Q e^{t}\right)$ of the $2 \mathrm{~d}$ period vector ${ }^{15}$ with double logarithmic behavior [43]. The index $\gamma$ runs over a basis of $H^{4}(X, \mathbb{Z}) \cap H^{2,2}(X)$. After normalization, the potentials $\mathcal{F}_{\gamma} \sim \Pi_{\gamma}^{\mathrm{ln}^{2}}$ have an expansion for large Kähler moduli of the

\footnotetext{
${ }^{15}$ I.e., the $2 \mathrm{~d}$ version of the solution vector eq. (2.5).
} 
form $[44,45]$

$$
\mathcal{F}_{\gamma}\left(Q e^{t}\right)=\mathcal{F}_{\gamma, c l}\left(Q e^{t}\right)+\sum_{\vec{d}>\overrightarrow{0}, n \geq 0} Q^{\vec{d}} \frac{\left(\sum_{j} d_{j} t_{j}\right)^{n}}{n !} \sum_{r \mid \vec{d}} \frac{\mathfrak{n}_{\gamma, \vec{d} / r}}{r^{2}}
$$

where the classical contribution $\mathcal{F}_{\gamma, c l}\left(Q e^{t}\right)$ is a degree two polynomial in $\ln \left(Q e^{t}\right)$. The invariants $\mathfrak{n}_{\gamma, \vec{d}}$ defined by this expansion are integral invariants associated with a 4-cycle $C_{\gamma} \in H_{4}(X, \mathbb{Z})$. The $h_{2,2}(X)$ potentials $\mathcal{F}_{\gamma}$ replace the Gromov-Witten potential (2.11) of the threefold case. They are related to the quantum corrected 3-point correlators for the operators $\phi_{\alpha}, \phi_{\beta} \in H^{1,1}(X, \mathbb{Z})$ and $\phi_{\gamma} \in H^{d-2, d-2}(X, \mathbb{Z})$ as

$$
C_{\alpha \beta \gamma}=\phi_{\alpha} \wedge \phi_{\beta} \wedge \phi_{\gamma}=\partial_{t_{\alpha}, t_{\beta}} \mathcal{F}_{\gamma}
$$

Computing correlators of the ordinary quantum K-theory for various Calabi-Yau $d$ folds, we find a very similar structure for the multi-cover contributions as for the threefold case in section 2.2. The $I$ function for a deformation $t=t_{a} \Phi_{a}$ has again the general form in eq. (2.8). Moreover, let $\Phi_{\gamma}=K_{\gamma}+\ldots$ be an element of the basis (2.4) with $K_{\gamma} \in H^{2,2}(X, \mathbb{Q})$ and

$$
\hat{F}_{\gamma}=\sum_{\vec{d}>0} Q^{\vec{d}}\left\langle\left\langle\frac{\Phi_{\gamma}}{1-q L}\right\rangle_{\vec{d}}, \quad \gamma=n_{K}+1, \ldots, n_{k}+h^{2,2}(X),\right.
$$

the quantum correlators associated with these elements. Then all our computations are consistent with the following conjectural expression of the correlators in terms of the invariants $\mathfrak{n}_{\gamma, \vec{d}}$ defined in eq. (3):

$$
\begin{aligned}
\hat{F}_{\gamma} & =p_{1, \gamma}+\frac{1}{(1-q)}\left[\mathcal{F}_{\gamma}\right]_{t^{n>1}}, a=1, \ldots n_{K}, \\
p_{1, \gamma} & =\sum_{\vec{d}>\overrightarrow{0}} Q^{\vec{d}} \sum_{r \mid \vec{d}} \mathfrak{n}_{\gamma, \vec{d} / r} \cdot\left\{(1-q) \frac{r\left(1-q^{r}\right)+q^{r}}{\left(1-q^{r}\right)^{2}}+\frac{1}{r}\left(\sum_{j} t_{j} d_{j}\right) \frac{1}{\left(1-q^{r}\right)}\right\}
\end{aligned}
$$

This is essentially the same formula as for the correlators $\hat{F}_{a}$ in the threefold case, with the replacement $d_{a} \mathfrak{n}_{\vec{d}}^{\mathrm{d}=3} \rightarrow \mathfrak{n}_{a, \vec{d}}^{\mathrm{d}>3}$.

As an example we consider the non-compact toric Calabi-Yau four-fold $X_{4}$ corresponding to the charge vectors

$$
l_{1}=(0 ;-3,1,1,1,0,0), \quad l_{2}=(0 ; 1,0,0,-1,1,-1) .
$$

The generalized $q$-hypergeometric series $\mathcal{I}$ for $X_{4}$, for zero effective Chern-Simons terms and with $\tilde{d}_{a}=d_{a}-\epsilon_{a}$, is

$$
\mathcal{I}=\sum_{d_{1}, d_{2} \geq 0} z_{1}^{\tilde{d}_{1}} z_{2}^{\tilde{d}_{2}} \frac{\Gamma_{q}\left(1-\epsilon_{1}\right)^{2} \Gamma_{q}\left(1-\epsilon_{2}\right) \Gamma_{q}\left(1+\epsilon_{2}\right) \Gamma_{q}\left(1+3 \epsilon_{1}-\epsilon_{2}\right) \Gamma_{q}\left(1-\epsilon_{1}+\epsilon_{2}\right)}{\Gamma_{q}\left(1+\tilde{d}_{1}\right)^{2} \Gamma_{q}\left(1+\tilde{d}_{2}\right) \Gamma_{q}\left(1-\tilde{d}_{2}\right) \Gamma_{q}\left(1-3 \tilde{d}_{1}+\tilde{d}_{2}\right) \Gamma_{q}\left(1+\tilde{d}_{1}-\tilde{d}_{2}\right)} .
$$

The correlators in ordinary quantum K-theory are obtained from the vortex sum $\mathcal{I}$ following the steps $\mathcal{I} \rightarrow I_{S Q K}(t) \rightarrow I(0) \rightarrow I_{Q K}(t)$ as outlined in section 2 for the threefold case. 


\begin{tabular}{|c|cccc|}
\hline & $Q_{2}^{1}$ & $Q_{2}^{2}$ & $Q_{2}^{3}$ & $Q_{2}^{4}$ \\
\hline$Q_{1}^{0}$ & -1 & $\frac{q^{2}-2}{(q+1)^{2}}$ & $\frac{2 q^{3}-3}{\left(q^{2}+q+1\right)^{2}}$ & $\frac{3 q^{4}-4}{(q+1)^{2}\left(q^{2}+1\right)^{2}}$ \\
$Q_{1}^{1}$ & 2 & 1 & 1 & 1 \\
$Q_{1}^{2}$ & -5 & $-\frac{2 q(3 q+4)}{(q+1)^{2}}$ & -3 & $-\frac{5 q^{2}+8 q+2}{(q+1)^{2}}$ \\
$Q_{1}^{3}$ & 32 & 21 & $\frac{2\left(9 q^{4}+16 q^{3}+27 q^{2}+18 q+12\right)}{\left(q^{2}+q+1\right)^{2}}$ & 20 \\
$Q_{1}^{4}$ & -286 & $-\frac{5\left(35 q^{2}+72 q+38\right)}{(q+1)^{2}}$ & -153 & $*$ \\
\hline
\end{tabular}

Table 1. 1-pt correlators for $X_{4}$ that compute disk invariants in the $q \rightarrow 1$ limit.

The two independent $q$-periods $F_{\gamma}$ can be chosen to have classical pieces $F_{3, c l}=\alpha_{3} t_{1}^{2}$ and $F_{4, c l}=\alpha_{4}\left(t_{1}+3 t_{2}\right)^{2}$ with constants $\alpha_{3,4}$; in this basis $F_{3}\left(t_{1}, Q_{1}\right)$ does not depend on $t_{2}$ and $Q_{2}$ and agrees with the $q$-period of the Calabi-Yau threefold $X_{3}$ for $\alpha_{3}=-\frac{1}{6}$. The 1-pt correlators obtained from $F_{4}$ with $\alpha_{4}=-\frac{1}{12}$ are listed in table 1 .

In the context of the $2 \mathrm{~d} / \mathrm{GW}$ correspondence, it is known [46] that the genus zero GW invariants of $X_{4}$ compute Ooguri-Vafa disk invariants for a certain family of Lagrangian branes $L$ in the threefold $X_{3}=\mathcal{O}(-3)_{\mathbb{P}^{2}}$ described in refs. [47-49], with $t_{1}$ and $t_{2}$ measuring the size of a sphere and a disk in the three-dimensional geometry $\left(X_{3}, L\right)$, respectively. Since the GW theory of $X_{4}$ can be obtained by sending the $S^{1}$ radius in the $3 \mathrm{~d}$ theory to zero, the $q \rightarrow 1$ limit of the $3 \mathrm{~d}$ QK invariants in table 1 also reproduces the $2 \mathrm{~d}$ disk invariants for the geometry $\left(X_{3}, L\right)$. It is natural to ask about an interpretation of the QK invariants in terms the geometry $\left(X_{3}, L\right)$, which would lift the $2 \mathrm{~d}$ open/closed duality between $\left(X_{3}, L\right)$ and $X_{4}$ to the $3 \mathrm{~d}$ theory. Such an interpretation should involve quantum K-theory on the moduli space of Riemann surfaces with boundary on the mathematical side and it would be interesting to study this further, perhaps along the lines of ref. [50], where a mathematical definition for the cohomological disk invariants has been given.

\section{Acknowledgments}

We would like to thank Bumsig Kim, Urmi Ninad and Yongbin Ruan for discussions. The work of P.M. is supported by the German Excellence Cluster Universe.

Open Access. This article is distributed under the terms of the Creative Commons Attribution License (CC-BY 4.0), which permits any use, distribution and reproduction in any medium, provided the original author(s) and source are credited.

\section{References}

[1] N. Nekrasov, Four Dimensional Holomorphic Theories, Ph.D. Thesis, (1996) [http://scgp.stonybrook.edu/people/faculty/bios/nikita-nekrasov].

[2] N.A. Nekrasov and S.L. Shatashvili, Supersymmetric vacua and Bethe ansatz, Nucl. Phys. Proc. Suppl. 192-193 (2009) 91 [arXiv:0901.4744] [INSPIRE]. 
[3] N.A. Nekrasov and S.L. Shatashvili, Quantization of Integrable Systems and Four Dimensional Gauge Theories, in Proceedings, 16th International Congress on Mathematical Physics (ICMP09), Prague, Czech Republic, 3-8 August 2009, pp. 265-289 (2009) [DOI:10.1142/9789814304634_0015] [arXiv:0908.4052] [INSPIRE].

[4] A. Givental, Permutation-equivariant quantum K-theory I. Definitions. Elementary K-theory of $\overline{\mathcal{M}}_{0, n} / S_{n}$, arXiv: 1508.02690 .

[5] A. Givental, Permutation-equivariant quantum K-theory II. Fixed point localization, arXiv: 1508.04374.

[6] A. Givental, Permutation-equivariant quantum K-theory III. Lefschetz' formula on $\overline{\mathcal{M}}_{0, n} / S_{n}$ and adelic characterization, arXiv:1508.06697.

[7] A. Givental, Permutation-equivariant quantum K-theory IV. $D_{q}$-modules arXiv:1509.00830.

[8] A. Givental, Permutation-equivariant quantum K-theory V. Toric q-hypergeometric functions, arXiv:1509.03903.

[9] A. Givental, Permutation-equivariant quantum K-theory VI. Mirrors, arXiv:1509.07852.

[10] A. Givental, Permutation-equivariant quantum K-theory VII. General theory, arXiv: 1510.03076.

[11] A. Givental, Permutation-equivariant quantum K-theory VIII. Explicit reconstruction, arXiv: 1510.06116.

[12] A. Givental, Permutation-equivariant quantum K-theory IX. Quantum Hirzebruch-Riemann-Roch in all genera, arXiv:1709.03180.

[13] A. Givental, Permutation-equivariant quantum K-theory X. Quantum Hirzebruch-Riemann-Roch in genus 0, arXiv:1710.02376.

[14] A. Givental, Permutation-equivariant quantum K-theory XI. Quantum Adams-Riemann-Roch, arXiv:1711.04201. [INSPIRE].

[15] H. Jockers and P. Mayr, A 3d Gauge Theory/Quantum k-theory Correspondence, arXiv: 1808.02040 [INSPIRE].

[16] E. Witten, Phases of $N=2$ theories in two-dimensions, Nucl. Phys. B 403 (1993) 159 [hep-th/9301042] [INSPIRE].

[17] D.R. Morrison and M.R. Plesser, Summing the instantons: Quantum cohomology and mirror symmetry in toric varieties, Nucl. Phys. B 440 (1995) 279 [hep-th/9412236] [INSPIRE].

[18] T. Dimofte, D. Gaiotto and S. Gukov, 3-Manifolds and 3d Indices, Adv. Theor. Math. Phys. 17 (2013) 975 [arXiv: 1112.5179] [INSPIRE].

[19] A. Kapustin and B. Willett, Wilson loops in supersymmetric Chern-Simons-matter theories and duality, arXiv:1302.2164 [INSPIRE].

[20] C. Beem, T. Dimofte and S. Pasquetti, Holomorphic Blocks in Three Dimensions, JHEP 12 (2014) 177 [arXiv:1211.1986] [InSPIRE].

[21] S. Cecotti, D. Gaiotto and C. Vafa, tt* geometry in 3 and 4 dimensions, JHEP 05 (2014) 055 [arXiv: 1312.1008] [INSPIRE].

[22] D. Gaiotto and P. Koroteev, On Three Dimensional Quiver Gauge Theories and Integrability, JHEP 05 (2013) 126 [arXiv:1304.0779] [INSPIRE]. 
[23] M. Bullimore, T. Dimofte, D. Gaiotto and J. Hilburn, Boundaries, Mirror Symmetry and Symplectic Duality in $3 d \mathcal{N}=4$ Gauge Theory, JHEP 10 (2016) 108 [arXiv:1603.08382] [INSPIRE].

[24] M. Aganagic and A. Okounkov, Elliptic stable envelopes, arXiv:1604.00423 [INSPIRE].

[25] M. Aganagic and A. Okounkov, Quasimap counts and Bethe eigenfunctions, Moscow Math. J. 17 (2017) 565 [arXiv: 1704.08746] [InSPIRE].

[26] P. Koroteev, P.P. Pushkar, A. Smirnov and A.M. Zeitlin, Quantum k-theory of Quiver Varieties and Many-Body Systems, arXiv:1705.10419 [INSPIRE].

[27] M. Bullimore, A. Ferrari and H. Kim, Twisted Indices of $3 d \mathcal{N}=4$ Gauge Theories and Enumerative Geometry of Quasi-Maps, JHEP 07 (2019) 014 [arXiv:1812.05567] [INSPIRE].

[28] B.H. Lian and S.-T. Yau, Mirror maps, modular relations and hypergeometric series 1, hep-th/9507151 [INSPIRE].

[29] R. Gopakumar and C. Vafa, M theory and topological strings. 1., hep-th/9809187 [INSPIRE].

[30] P. Candelas, X. De La Ossa, A. Font, S.H. Katz and D.R. Morrison, Mirror symmetry for two parameter models. 1., Nucl. Phys. B 416 (1994) 481 [hep-th/9308083] [INSPIRE].

[31] S. Hosono, A. Klemm, S. Theisen and S.-T. Yau, Mirror symmetry, mirror map and applications to Calabi-Yau hypersurfaces, Commun. Math. Phys. 167 (1995) 301 [hep-th/9308122] [INSPIRE].

[32] A. Gadde, S. Gukov and P. Putrov, Walls, Lines and Spectral Dualities in 3d Gauge Theories, JHEP 05 (2014) 047 [arXiv: 1302.0015] [INSPIRE].

[33] Y. Yoshida and K. Sugiyama, Localization of $3 d \mathcal{N}=2$ Supersymmetric Theories on $S^{1} \times D^{2}$, arXiv:1409.6713 [inSPIRE].

[34] D.A. Cox and S. Katz, Mirror symmetry and algebraic geometry, Mathematical Surveys and Monographs, vol. 68, American Mathematical Society, Providence, RI (1999).

[35] Y. Ruan and M. Zhang, The level structure in quantum K-theory and mock theta functions, arXiv: 1804.06552.

[36] S. Hosono, A. Klemm, S. Theisen and S.-T. Yau, Mirror symmetry, mirror map and applications to complete intersection Calabi-Yau spaces, Nucl. Phys. B 433 (1995) 501 [hep-th/9406055] [INSPIRE].

[37] A. Givental, Explicit reconstruction in quantum cohomology and K-theory, Ann. Fac. Sci. Toulouse Math. 25 (2016) 419 [arXiv:1506.06431].

[38] A. Givental and V. Tonita, The Hirzebruch-Riemann-Roch theorem in true genus-0 quantum $k$-theory, arXiv:1106.3136 [INSPIRE].

[39] Y.-P. Lee, Quantum K-theory. I. Foundations, Duke Math. J. 121 (2004) 389 [math/0105014].

[40] P.S. Aspinwall and D.R. Morrison, Topological field theory and rational curves, Commun. Math. Phys. 151 (1993) 245 [hep-th/9110048] [INSPIRE].

[41] A. Givental, On the WDVV equation in quantum K-theory, Michigan Math. J. 48 (2000) 295 [math/0003158].

[42] H. Iritani, T. Milanov and V. Tonita, Reconstruction and Convergence in Quantum K-Theory via Difference Equations, Int. Math. Res. Not. (2015) 2887 [arXiv:1309.3750] [INSPIRE]. 
[43] B.R. Greene, D.R. Morrison and M.R. Plesser, Mirror manifolds in higher dimension, Commun. Math. Phys. 173 (1995) 559 [hep-th/9402119] [INSPIRE].

[44] P. Mayr, Mirror symmetry, $N=1$ superpotentials and tensionless strings on Calabi-Yau four folds, Nucl. Phys. B 494 (1997) 489 [hep-th/9610162] [INSPIRE].

[45] A. Klemm, B. Lian, S.S. Roan and S.-T. Yau, Calabi-Yau fourfolds for M-theory and F-theory compactifications, Nucl. Phys. B 518 (1998) 515 [hep-th/9701023] [INSPIRE].

[46] P. Mayr, $N=1$ mirror symmetry and open/closed string duality, Adv. Theor. Math. Phys. $\mathbf{5}$ (2002) 213 [hep-th/0108229] [INSPIRE].

[47] H. Ooguri and C. Vafa, Knot invariants and topological strings, Nucl. Phys. B 577 (2000) 419 [hep-th/9912123] [INSPIRE].

[48] M. Aganagic and C. Vafa, Mirror symmetry, D-branes and counting holomorphic discs, hep-th/0012041 [INSPIRE].

[49] M. Aganagic, A. Klemm and C. Vafa, Disk instantons, mirror symmetry and the duality web, Z. Naturforsch. A 57 (2002) 1 [hep-th/0105045] [INSPIRE].

[50] S.H. Katz and C.-C.M. Liu, Enumerative geometry of stable maps with Lagrangian boundary conditions and multiple covers of the disc, Adv. Theor. Math. Phys. 5 (2001) 1 [math/0103074] [INSPIRE]. 